\title{
SCIENTIFIC REPORTS

\section{SLPI - a Biomarker of Acute Kidney Injury after Open and Endovascular Thoracoabdominal Aortic Aneurysm (TAAA) Repair}

Luisa Averdunk ${ }^{1}$, Marcia V. Rückbeil ${ }^{2}$, Alexander Zarbock ${ }^{3}$, Lukas Martin ${ }^{1}{ }^{1}$, Gernot Marx ${ }^{1}$, Houman Jalaie ${ }^{4}$, Michael J. Jacobs ${ }^{4}$, Christian Stoppe ${ }^{1,5}$ \& Alexander Gombert $\mathbb{B}^{4,5^{*}}$

Acute kidney injury (AKI) is a relevant complication following thoracoabdominal aortic aneurysm repair (TAAA). Biomarkers, such as secretory leucocyte peptidase inhibitor (SLPI), may enable a more accurate diagnosis. In this study, we tested if SLPI measured in serum is an appropriate biomarker of AKI after TAAA repair. In a prospective observational single-center study including 33 patients $(51.5 \%$ women, mean age $63.0 \pm 16.2$ years) undergoing open and endovascular aortic aneurysm repair in 2017, SLPI was measured peri-operatively (until $72 \mathrm{~h}$ after surgery). After surgery, the postoperative complications AKI, as defined according to the KDIGO diagnostic criteria, sepsis, death, MACE (major cardiovascular events) and, pneumonia were assessed. In a subgroup analysis, patients with preexisting kidney disease were excluded. Of 33 patients, $51.5 \%(n=17)$ of patients developed AKI. Twelve hours after admission to the intensive care unit (ICU), SLPI serum levels were significantly increased in patients who developed AKI. Multivariable logistic regression revealed a significant association between SLPI 12 hours after admission to ICU and AKI $(P=0.0181, \mathrm{OR}=1.055,95 \% \mathrm{Cl}=1.009-1.103)$. The sensitivity of SLPI for AKI prediction was $76.47 \%(95 \% \mathrm{Cl}=50.1-93.2)$ and the specificity was $87.5 \%(95 \% \mathrm{Cl}=$ 61.7-98.4) with an AUC $=0.838(95 \% \mathrm{Cl}=0.7-0.976)$ for an optimal cut-off $70.03 \mathrm{ng} / \mathrm{ml} 12$ hours after surgery. In patients without pre-existing impaired renal function, an improved diagnostic quality of SLPI for AKI was observed (Sensitivities of 45.45-91.67\%, Specificities of 77.7-100\%, AUC $=0.716-0.932$ ). There was no association between perioperative SLPI and the incidence of sepsis, death, MACE (major cardiovascular events), pneumonia. This study suggests that SLPI might be a post-operative biomarker of AKI afterTAAA repair, with a superior diagnostic accuracy for patients without preexisting impaired renal function.

Open and endovascular repair of thoracoabdominal aortic aneurysm (TAAA) is related to a high risk of postoperative complications. With an incidence ranging between 13 and 42\%, acute kidney injury (AKI) is one of the most common complications and closely associated with increased mortality and cardiovascular morbidity ${ }^{1-3}$. The early detection of impaired kidney function and other organ dysfunctions may enable an immediate start of specific treatment bundles. The diagnosis of AKI is mainly based on patients' urine output and serum creatinine levels. Serum creatinine is an established, yet controversial biomarker due to its delayed increase and low sensitivity for the detection of an impaired kidney function ${ }^{4-6}$. In this context, the necessity of clinically available early and reliable biomarkers of AKI becomes evident.

Secretory leucocyte peptidase inhibitor (SLPI) is a protease inhibitor and regulator of innate and adaptive immunity ${ }^{7}$. It is synthesized predominantly in immune and epithelial cells of mucosal surfaces, such as the pancreas and kidney ${ }^{8}$. Elevated serum SLPI levels have been observed in acute and chronic inflammatory conditions

\footnotetext{
${ }^{1}$ Department of Intensive Care and Intermediate Care, University Hospital Aachen, RWTH Aachen University, Aachen, Germany. ${ }^{2}$ Department of Medical Statistics, University Hospital Aachen, RWTH Aachen University, Aachen, Germany. ${ }^{3}$ Department of Anesthesiology, Intensive Care, University Hospital Muenster, Münster, Germany. ${ }^{4}$ European Vascular Center Aachen-Maastricht, University Hospital Aachen, RWTH Aachen University, Aachen, Germany. ${ }^{5}$ These authors contributed equally: Christian Stoppe and Alexander Gombert. *email: agombert@ ukaachen.de
} 
such as acute lung injury ${ }^{9,10}$. In the setting of oxidative stress, SLPI seems to have antioxidant and cytoprotective properties $^{11,12}$. In a murine model of experimental ischemic AKI, Macrophage Migration Inhibitory Factor- 2 (MIF-2) was suggested to exert kidney protection by upregulation of SLPI expression ${ }^{13}$.

In human kidney biopsies taken from patients with early post-transplant AKI after kidney transplantation, whole-genome mRNA profiling revealed a significant (15-fold) upregulation of SLPI mRNA expression compared to patients not affected by post-transplant AKI. Additionally, patients with post-transplant AKI showed significantly increased SLPI plasma and urine SLPI when compared with patients without $\mathrm{AKI}^{14}$. In a recent study, we found SLPI to be a candidate biomarker for the early diagnosis of AKI after cardiac surgery ${ }^{15}$. However, the performance of SLPI as a biomarker of AKI in the setting of TAAA repair has not yet been investigated.

The aim of this study was to evaluate the role of SLPI as a potential biomarker for the prediction of postoperative AKI in patients undergoing complex open and endovascular TAAA repair.

\section{Methods}

Study design. The internal review board of the University Hospital Aachen (EK004/14) authorized this study. We performed this study in accordance with the Declaration of Helsinki. Preoperatively, informed consent was obtained from all subjects.

If an elective open or endovascular TAAA repair, defined according to the Crawford classification, was planned, patients were eligible for inclusion ${ }^{16}$.

Patients undergoing TAAA repair between January and December 2017 were consecutively screened. After excluding patients treated as emergency cases, the following exclusion criteria have been applied: Chronic kidney disease with dialysis treatment, age below 18 years, pregnancy and immunosuppressive medication. 33 patients were included in this prospective study. Medical history and physiological parameters were taken from medical records and electronic bedside flow charts (IntelliSpace Critical Care and Anesthesia; Philips Healthcare, Andover, Massachusetts, USA). Serum samples were collected before surgery, after admission to the intensive care unit (ICU), as well as during early follow up on ICU $(12,24,48$, and 72 hours). AKI was defined according to the KDIGO criteria ${ }^{17}$ based on serum creatinine levels and 24-hour urine output detection during the first 72 hours after surgery. Baseline creatinine was defined as the lowest pre-intervention value 24 hours before surgery.

SLPI measurement. Serum samples were collected one day before the TAAA repair, after admission to the ICU as well as 12, 24, 48 and 72 hours afterwards. These samples were centrifuged with $3000 \mathrm{rpm}$ for ten minutes, afterwards supernatants were transferred to cryotubes and stored at $-80^{\circ} \mathrm{C}$ aaccording to the manufacturer's advice (R\&D systems, Minneapolis, MN). Serum levels of SLPI were measured by ELISA. The average coefficient of variation (CV) between duplicates was $9.8 \%$ (intra-assay $\mathrm{CV}$ ) and the average inter-assay coefficient was $13.4 \%$.

Surgery. As published before, the protocol for open TAAA repair included aortic cross-clamping, extracorporal circulation with distal aortic perfusion, and visceral perfusion using selective perfusion catheters ${ }^{18-20}$. Renal perfusion was realized by using $4^{\circ} \mathrm{C}$ tempered Custodiol ${ }^{\circledR}$ (Dr. Franz Köhler Chemie, Austria) to avoid ischemic organ damage ${ }^{21}$. To avoid renal failure, contrast agent was used carefully, leading to a mean application of $65 \pm 17 \mathrm{ml}$ per endovascular procedure. Furthermore, we applied one fourth of the standard dose for kidney angiography ${ }^{22}$.

Endpoints. The assessment of the kinetics of serum SLPI and its applicability as a potential biomarker of AKI after TAAA repair was the motivation for this study. In a subgroup analysis, patients with pre-operative chronic kidney failure (defined as preoperative serum creatinine $>1.25 \mathrm{mg} / \mathrm{dl}$ according to cut-off used in the Cleveland clinic foundation score ${ }^{23}$ were excluded, to select those patients with physiological preoperative kidney function and reduce the heterogeneity of the cohort. As secondary endpoints, the association of serum SLPI with the following postoperative adverse events was analyzed: Sepsis, death, MACE (major cardiovascular events), pneumonia. Pneumonia and tracheotomy were defined according to the guidelines of the American Thoracic Society or the Belgian Society of Pneumology, respectively ${ }^{24,25}$. Spinal cord ischemia was defined as postoperative paraplegia or paraparesis ${ }^{20}$. Major cardiovascular events (MACE) included myocardial infarction, acute heart failure and ventricular tachycardia; all defined according to current guidelines ${ }^{26-28}$. Sepsis was defined according to the guidelines of the German Sepsis Society ${ }^{29}$ : Fever above $38^{\circ} \mathrm{C}$ or hypothermia below $36^{\circ} \mathrm{C}$, tachycardia with a heart rate above 90 beats per minute, tachypnea with a respiratory rate above 20 per minute or a leukocytosis $\left(\geq 12000 / \mathrm{mm}^{3}\right)$ or leucopenia $\left(\leq 4000 / \mathrm{mm}^{3}\right)$. For patients and time points when clinical data were available, we additionally correlated serum SLPI with the inflammatory markers CRP, PCT, IL-6 and white blood cell count measured on ICU.

Statistics. The continuous variables are expressed as median with lower and upper quartile (Q1-Q3) in case of heavily skewed data or as means \pm standard deviation (SD). Categorical variables are shown as absolute frequencies and percentages. The time course of perioperative serum SLPI is visualized in boxplots. In a linear model with unstructured covariance structure to illustrate the correlation between repeated measurements within each patient we tested for differences in SLPI between open and endovascular surgery.

The association between the occurrence of an AKI and other clinical outcomes (e.g. pneumonia) was assessed using Fisher's exact test. Firth's bias correction was used in an univariable logistic regression model to identify associations between baseline or operational characteristics and the development of an AKI. Associations between the development of an AKI (dependent variable) and serum SLPI were likewise assessed using a univariable logistic regression model with Firth's bias correction. The time point with the best association (SLPI 12 hours after ICU) was selected as an independent variable for a multivariable logistic regression analysis. The model further included the type of surgery and all patient characteristics from Table 1 that had a $P$-value of at most 0.2 in 


\begin{tabular}{|c|c|c|c|c|}
\hline \multirow[b]{3}{*}{ Characteristic } & \multirow{3}{*}{$\begin{array}{l}\text { All patients } \\
(\mathrm{N}=33)\end{array}$} & \multicolumn{2}{|c|}{ Acute kidney injury (AKI) } & \multirow[b]{3}{*}{$P$-value ${ }^{\text {a }}$} \\
\hline & & No & Yes & \\
\hline & & $(\mathrm{N}=16)$ & $(\mathrm{N}=17)$ & \\
\hline \multicolumn{5}{|l|}{ Demographics } \\
\hline Age, years & $63.0 \pm 16.2$ & $65.4 \pm 15.1$ & $60.8 \pm 17.3$ & 0.4724 \\
\hline Sex (male) & $16(48.48 \%)$ & $10(62.50 \%)$ & $6(35.29 \%)$ & 0.1392 \\
\hline $\mathrm{BMI}, \mathrm{kg} / \mathrm{m}^{2}$ & $25.4 \pm 5.0$ & $25.9 \pm 5.4$ & $24.9 \pm 4.8$ & 0.6156 \\
\hline Current smokers & $12(36.36 \%)$ & $6(37.50 \%)$ & $6(35.29 \%)$ & 0.7638 \\
\hline \multicolumn{5}{|l|}{ Comorbidities } \\
\hline Chronic kidney disease & $5(15.15 \%)$ & $3(18.75 \%)$ & $2(11.76 \%)$ & 0.8403 \\
\hline Coronary heart disease & $14(42.42 \%)$ & $9(56.25 \%)$ & $5(29.41 \%)$ & 0.1471 \\
\hline Diabetes mellitus & $6(18.18 \%)$ & $2(12.50 \%)$ & $4(23.53 \%)$ & 0.4807 \\
\hline Hypertension & $23(69.7 \%)$ & $14(87.50 \%)$ & $9(52.94 \%)$ & 0.0575 \\
\hline COPD & $13(39.39 \%)$ & $8(50 \%)$ & $5(29.41 \%)$ & 0.2593 \\
\hline Connective tissue disease (Marfan syndrome) & $5(15.15 \%)$ & $1(6.25 \%)$ & $4(23.53 \%)$ & 0.2609 \\
\hline pAVK & $4(12.12 \%)$ & $2(12.50 \%)$ & $2(11.76 \%)$ & 0.9503 \\
\hline Maximum aortic diameter, $\mathrm{cm}$ & $6.6 \pm 1.3$ & $6.5 \pm 1.4$ & $6.7 \pm 1.1$ & 0.6691 \\
\hline \multicolumn{5}{|l|}{ Marker at baseline } \\
\hline Hemoglobin, g/dL & $12.9 \pm 1.9$ & $12.9 \pm 2.3$ & $12.8 \pm 1.5$ & 0.9156 \\
\hline Serum creatinine, $\mathrm{mg} / \mathrm{dL}$ & $1.1 \pm 0.4$ & $1.2 \pm 0.4$ & $1.0 \pm 0.3$ & 0.2413 \\
\hline \multicolumn{5}{|l|}{ Type of TAAA } \\
\hline TAAA 1 & $5(15.15 \%)$ & $3(18.75 \%)$ & $2(11.76 \%)$ & \multirow{5}{*}{0.3272} \\
\hline TAAA 2 & $7(21.21 \%)$ & $2(12.50 \%)$ & $4(29.41 \%)$ & \\
\hline TAAA 3 & $7(21.21 \%)$ & $1(6.25 \%)$ & $6(35.29 \%)$ & \\
\hline TAAA 4 & $10(30.3 \%)$ & $7(43.75 \%)$ & $3(17.65 \%)$ & \\
\hline TAAA 5 & $4(12.12 \%)$ & $3(18.75 \%)$ & $1(5.88 \%)$ & \\
\hline
\end{tabular}

Table 1. Patient characteristics in the entire collective and by AKI. Continuous data is reported as mean $\pm \mathrm{SD}$, categorical data as absolute and relative frequencies. ${ }^{a}$ Compared using a logistic regression model with Firth's bias correction.

the univariable logistic regression model as independent variables. For AKI these were SLPI 12 hours after ICU, sex, the presence of a coronary heart disease, hypertension, and the type of surgery.

The diagnostic quality of SLPI for predicting AKI was assessed using receiver operating characteristic curves (ROC curves). Sensitivity (Se), specificity (Sp), positive and negative likelihood ratio (LR+ and LR-), area under the curve (AUC) and the optimal cut-off value according to the Youden index are reported together with the ROC curves. Additional analyses were performed in the subgroup of patients without pre-existing impaired renal function.

The association between SLPI and other outcomes (sepsis, death, MACE, pneumonia) is shown in boxplots in the supplement. Associations were tested using a logistic regression model with the outcome as dependent variable and using Firth's bias correction. The level of significance was set at $5 \%$. No adjustments were made for multiple comparisons due to the exploratory nature of this study. Statistical analyses were performed using SAS software version 9.4 (SAS Institute, Cary NC, USA) and R, version 3.5.1 ${ }^{30}$.

\section{Results}

The mean patients' age was $63 \pm 1.26$ years, $51.5 \%$ were women. Demographical and baseline information as well as procedural details can be found in Tables 1 and 2. Seventeen patients (51.5\%) developed postoperative AKI as diagnosed according to the KDIGO classification criteria. From these seventeen patients, ten (58.8\%) were classified as KDIGO 1, two (11.7\%) as KDIGO 2 and five patients (29.4\%) as KDIGO 3. All patients with AKI fulfilled the diagnostic criteria of a rise in serum creatinine, but only six patients showed a significantly reduced urine output (KDIGO 1: $\mathrm{N}=1, \mathrm{KDIGO} 2: \mathrm{N}=1, \mathrm{KDIGO} 3: \mathrm{N}=4$ ). All details can be found in Table 3 .

Patients suffering from AKI had an increased risk of pneumonia (29.41\% vs. 6.25\%), sepsis ( $29.41 \%$ vs. $6.25 \%)$, and in-hospital mortality ( $29.41 \%$ vs. $6.25 \%)$.

Association of serum SLPI with AKI and postoperative adverse events. SLPI serum levels showed a biphasic course with a significant decline from the day before surgery to admission to ICU after surgery ( 57 vs. $32 \mathrm{ng} / \mathrm{ml}, P=0.0002$ ) and a significant increase during the first 12 hours (Table 4). Serum SLPI remained high until 48 hours and reached baseline values at 72 hours after admission to ICU. No significant differences in serum SLPI were observed between patients undergoing open or endovascular TAAA repair (linear mixed model, $P=$ 0.7691, Fig. 1).

Twelve hours after admission to ICU, patients who developed AKI displayed significantly higher serum SLPI (AKI: $P=0.0058$ ) (Table 4, Fig. 2). In the subgroup of patients without pre-existing renal function impairment 


\begin{tabular}{|c|c|c|c|c|}
\hline \multirow[b]{3}{*}{ Characteristic } & \multirow{3}{*}{$\begin{array}{l}\text { All patients } \\
(\mathrm{N}=33)\end{array}$} & \multicolumn{2}{|c|}{ Acute kidney injury (AKI) } & \multirow[b]{3}{*}{$P$-value } \\
\hline & & \multirow{2}{*}{$\begin{array}{l}\text { No } \\
(\mathrm{N}=16)\end{array}$} & \multirow{2}{*}{$\begin{array}{l}\text { Yes } \\
(\mathrm{N}=17)\end{array}$} & \\
\hline & & & & \\
\hline \multicolumn{5}{|l|}{ Surgery } \\
\hline Endovascular surgery & $19(57.6 \%)$ & $12(75 \%)$ & $7(41.18 \%)$ & 0.0707 \\
\hline Open surgery & $14(42.4 \%)$ & $4(25 \%)$ & $10(58.82 \%)$ & \\
\hline Operation time, min & $374.3 \pm 111$ & $329.7 \pm 101.3$ & $416.3 \pm 105.7$ & 0.0466 \\
\hline ICU ventilation time, min & $835(300-1571)$ & $350(0-817.5)$ & $1149(965-2147)$ & 0.1381 \\
\hline Total ventilation time, min & $1410(960-2505)$ & $1020(582.5-1410)$ & $1940(1410-4865)$ & 0.0491 \\
\hline Stay on ICU, days & $4(3-5)$ & $3(1.5-5)$ & $5(4-9)$ & 0.0595 \\
\hline In-hospital stay, days & $26(11-35)$ & $20.5(10-33)$ & $28(19-38)$ & 0.3621 \\
\hline Blood transfusion (blood bags) & $8(4-15)$ & $5(2-7)$ & $13(9-27)$ & 0.1290 \\
\hline
\end{tabular}

Table 2. Operational characteristics in the entire collective and by AKI. Continuous data is reported as mean \pm SD or median (Q1-Q3) in case of heavily skewed data, categorical data as absolute and relative frequencies. ${ }^{a}$ Compared using a logistic regression model with Firth's bias correction where skewed characteristics were logarithmically transformed.

\begin{tabular}{|c|c|c|c|c|}
\hline \multirow[b]{3}{*}{ Outcome } & \multirow{3}{*}{\begin{tabular}{|l} 
All patients \\
$(\mathrm{N}=33)$
\end{tabular}} & \multicolumn{2}{|c|}{ Acute kidney injury (AKI) } & \multirow[b]{3}{*}{$P$-value } \\
\hline & & \multirow{2}{*}{\begin{tabular}{|l} 
No \\
$(\mathrm{N}=16)$ \\
\end{tabular}} & \multirow{2}{*}{\begin{tabular}{|l} 
Yes \\
$(\mathrm{N}=17)$ \\
\end{tabular}} & \\
\hline & & & & \\
\hline Pneumonia & $6(18.18 \%)$ & $1(6.25 \%)$ & $5(29.41 \%)$ & 0.1748 \\
\hline Tracheotomy & $4(12.12 \%)$ & $1(6.25 \%)$ & $3(17.65 \%)$ & 0.6012 \\
\hline Spinal cord ischemia & $3(9.09 \%)$ & $2(12.50 \%)$ & $1(5.88 \%)$ & 0.6012 \\
\hline Major cardiovascular events (MACE) & $10(30.30 \%)$ & $3(18.75 \%)$ & $7(41.18 \%)$ & 0.2587 \\
\hline Sepsis & $6(18.18 \%)$ & $1(6.25 \%)$ & $5(29.41 \%)$ & 0.1748 \\
\hline In-hospital mortality & $6(18.18 \%)$ & $1(6.25 \%)$ & $5(29.41 \%)$ & 0.1748 \\
\hline
\end{tabular}

Table 3. Incidence of postoperative complications in the entire collective and by AKI. Data is reported as

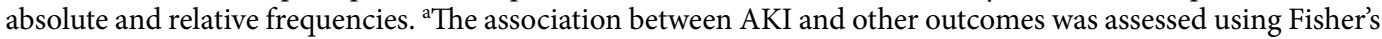
exact test.

(preoperative creatinine $\leq 1.25 \mathrm{mg} / \mathrm{dl}$ ), significantly increased serum SLPI was observed 12 and 24 hours post-interventionally in patients with AKI (Fig. 3). Besides, serum SLPI 12 hours after surgery was negatively correlated with urine output during the first 24 hours after surgery (Spearman coefficient $=-0.48$ and $95 \% \mathrm{CI}=$ $-0.71-0.16)$. Serum SLPI did not differ between patients affected by sepsis, MACE, death, or pneumonia compared to patients not affected by these adverse events (Figs. S1-4). Serum SLPI was significantly correlated with procalcitonin 24 and 72 hours after surgery, but did not show a significant correlation with CRP, IL-6 and white blood cells at any time point analyzed (PCT $24 \mathrm{~h}: P=0.018, \mathrm{R}^{2}=0.288$; PCT $72 \mathrm{~h}: P=0.025, \mathrm{R}^{2}=0.226$, Figure S5).

Multivariable logistic regression model. Next, we applied a multivariable logistic regression analysis to characterize the prognostic value of serum SLPI for AKI (Table 5). In this model, SLPI 12 hours after admission to ICU was significantly associated with the occurrence of an $\mathrm{AKI}(\mathrm{OR}=1.055,95 \% \mathrm{CI}=1.009-1.103, P=0.0181)$. None of the other independent variables showed a statistically significant association with AKI.

Diagnostic accuracy of SLPI as a predictor of AKI. The analysis by Receiver Operation Characteristics (ROC) curves revealed an adequate predictive accuracy of SLPI to detect AKI 12 and 24 hours after admission to ICU (for the optimal cut-off $70.03 \mathrm{ng} / \mathrm{ml}$ at 12 hours: Sensitivity $76.47 \%, 95 \% \mathrm{CI}=50.1-93.2$, Specificity $87.5 \%$, $95 \% \mathrm{CI}=61.7-98.4$, AUC $=0.838,95 \% \mathrm{CI}=0.7-0.976$; for the optimal cut-off of $56.33 \mathrm{ng} / \mathrm{ml}$ at 24 hours: Sensitivity $75 \%, 95 \%$ CI $=47.6-92.7 \%$, Specificity $71.4 \%$, 95\% CI $=41.9-91.6 \%$ AUC $=0.723,95 \%$ CI $=0.523-$ 0.923, Table 6, Fig. 4).

Diagnostic accuracy of SLPI in a subgroup without preoperative impaired renal function. The diagnostic performance of SLPI to predict AKI was improved in the subgroup of patients without pre-existing renal functional impairment (e.g. for 12 hours after admission to ICU: AUC $=0.932,95 \%$ CI $=0.83-1$ ) (Table 7, Fig. 4).

\section{Discussion}

Mortality and morbidity after open and endovascular TAAA Repair remain high ${ }^{31,32}$. In our observational study including TAAA patients, AKI was the most frequent complication after surgery and showed a crucial association with additional severe complications. 


\begin{tabular}{|c|c|c|c|c|}
\hline \multicolumn{5}{|l|}{ All patients } \\
\hline \multirow[b]{2}{*}{ Time } & \multirow[b]{2}{*}{ All patients $(\mathrm{N}=33)$} & \multicolumn{3}{|c|}{ Acute kidney injury (AKI) } \\
\hline & & No $(\mathrm{N}=16)$ & Yes $(\mathrm{N}=17)$ & $P$-value ${ }^{\text {a }}$ \\
\hline Baseline & $51.85(43.05-75.12)$ & $61.11(43.36-80.59)$ & $50.45(38.89-73.66)$ & 0.4342 \\
\hline Admission to ICU & $35.13(20.63-53.36)$ & $33.28(21.12-35.80)$ & $48.03(20.63-56.43)$ & 0.3807 \\
\hline $12 \mathrm{~h}$ after ICU & $64.00(42.51-84.59)$ & $45.46(35.91-61.04)$ & $84.21(70.03-101.93)$ & 0.0058 \\
\hline $24 \mathrm{~h}$ after ICU & $58.15(40.77-96.12)$ & $44.17(36.54-61.19)$ & 71.47 (51.90-98.59) & 0.3735 \\
\hline $48 \mathrm{~h}$ after ICU & $62.90(46.96-93.05)$ & $51.01(43.54-64.74)$ & $87.64(61.05-100.12)$ & 0.2077 \\
\hline $72 \mathrm{~h}$ after ICU & $50.40(32.03-67.25)$ & $40.60(32.03-54.57)$ & $54.21(33.71-69.24)$ & 0.2032 \\
\hline \multicolumn{5}{|c|}{ Patients with serum creatinine at baseline $\leq 1.25 \mathrm{mg} / \mathrm{dL}$} \\
\hline \multicolumn{5}{|c|}{ Acute kidney injury (AKI) } \\
\hline Time & All patients $(\mathrm{N}=22)$ & No $(\mathbf{N}=9)$ & Yes $(\mathrm{N}=13)$ & $P$-value ${ }^{a}$ \\
\hline Baseline & $51.33(36.29-74.36)$ & 45.08 (43.52-74.36) & $52.21(36.29-73.66)$ & 0.8365 \\
\hline Admission to ICU & $34.86(20.58-54.40)$ & $26.86(20.58-40.50)$ & $43.67(19.97-55.42)$ & 0.9141 \\
\hline $12 \mathrm{~h}$ after ICU & $52.93(37.13-84.21)$ & $36.23(33.47-45.64)$ & $75.80(57.02-89.25)$ & 0.0240 \\
\hline $24 \mathrm{~h}$ after ICU & $49.04(36.66-87.77)$ & $36.66(32.89-40.77)$ & 78.51 (51.64-98.59) & 0.0339 \\
\hline $48 \mathrm{~h}$ after ICU & 67.99 (45.96-96.58) & $46.96(37.56-51.01)$ & $90.84(71.24-105.57)$ & 0.0660 \\
\hline $72 \mathrm{~h}$ after ICU & $36.33(30.87-68.81)$ & $33.90(29.38-39.32)$ & $55.00(31.48-70.63)$ & 0.1288 \\
\hline
\end{tabular}

Table 4. SLPI in $\mathrm{ng} / \mathrm{ml}$ measured at different times in the entire collective and by AKI. Data is reported as

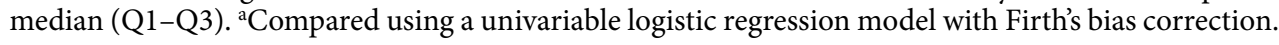

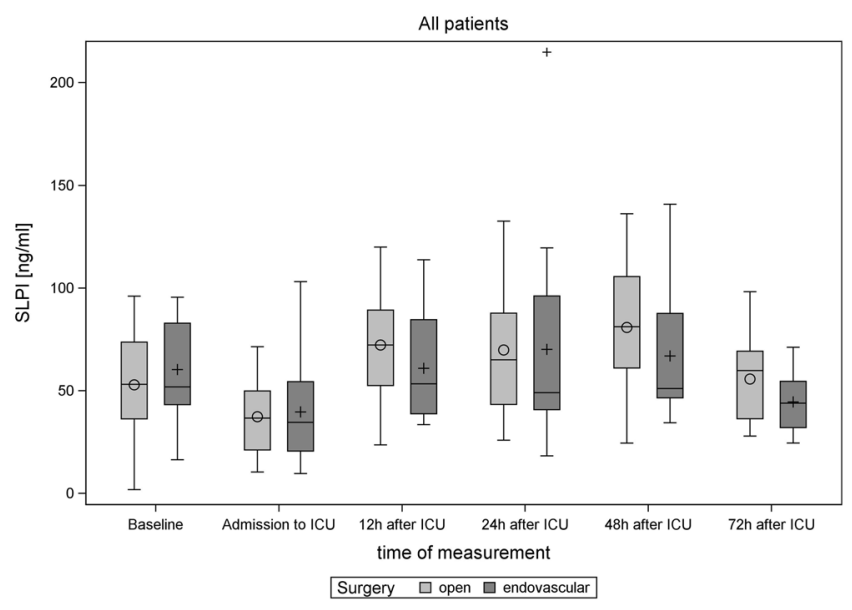

Figure 1. Boxplots illustrating SLPI levels before and after surgery in patients undergoing endovascular and open TAAA repair. There was no statistically significant difference in serum SLPI levels between patients undergoing open or endovascular TAAA repair (linear mixed model, $P=0.7691$ ).

Previous studies demonstrated that serum creatinine as an indirect marker of impaired renal function is inappropriate to detect early stages of $\mathrm{AKI}^{5,6}$ As treatment options of AKI are limited, the early identification of AKI by biomarkers and the immediate initiation of treatment are urgently needed to decrease the incidence and clinical consequences of AKI. The KDIGO clinical practice guideline recommends different preventive measures for the treatment of AKI. Next to the eradication of potentially nephrotoxic agents, an appropriate fluid management is important to prevent AKI in critically ill patients ${ }^{33}$. Besides, an early initiation of renal replacement therapy was suggested to improve the long-term survival of patients who suffered from $\mathrm{AKI}^{34}$.

To date, only a few biomarkers of postoperative complications have been investigated in the setting of TAAA. Recently, the diagnostic relevance of urinary neutrophile gelatinase associated lipocalin (NGAL) for postoperative AKI requiring dialysis was evaluated ${ }^{35}$. Up to now the quantification of NGAL has failed to reliably predict $\mathrm{AKI}^{35}$. One potential reason for why the postoperative detection of NGAL in the urine has not yet been put into clinical practice might be the circumstance, that urine samples are not routinely drawn for clinical chemistry analysis. Thus, it might be beneficial to identify appropriate kidney injury markers in the serum, which would be more feasible to be established as a routine diagnostic biomarker for AKI.

SLPI $(12 \mathrm{kDa})$ is a serine protease inhibitor and is expressed by macrophages, neutrophils, and many epithelial cells including the lung and kidney ${ }^{36}$.

By inhibiting neutrophil elastase, SLPI protects proteins from digestion ${ }^{37}$. Besides, SLPI was shown to inhibit the proinflammatory transcription factor $\mathrm{NF} k \mathrm{~B}$ and excessive inflammatory responses ${ }^{38}$. Apart from its anti-inflammatory functions, SLPI may control the growth of bacteria and fungi in a charge-dependent manner 


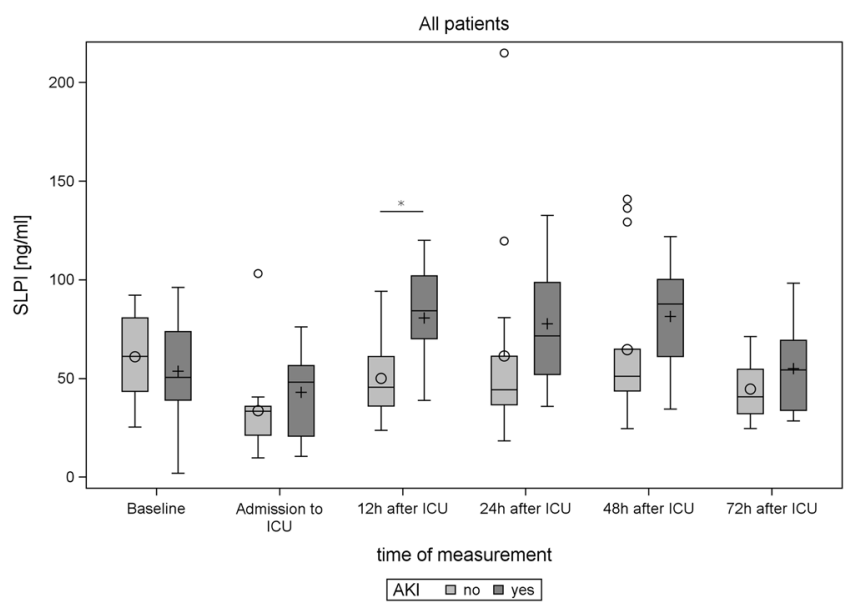

Figure 2. Boxplots illustrating SLPI levels before and after surgery in AKI versus non-AKI patients. Significant differences $(P$-values $<0.05$ in the corresponding analysis from Table 4$)$ are indicated by $*$.

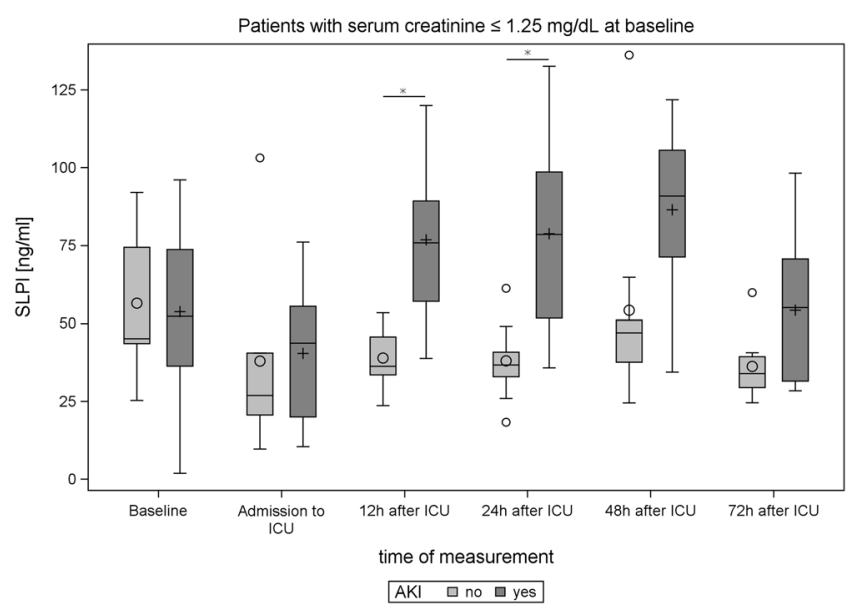

Figure 3. Boxplots of the subgroup of patients without pre-existing renal functional impairment illustrating the SLPI-levels before and after surgery in AKI versus non-AKI patients. Significant differences $(P$-values $<0.05$ in the corresponding analysis from Table 4 ) are indicated by $*$.

\begin{tabular}{|l|l|l|}
\hline Independent variable & $\begin{array}{l}\text { Odds ratio }[95 \% \\
\text { Confidence interval }]\end{array}$ & $\boldsymbol{P}$-value \\
\hline Sex (male vs. female) & $0.193[0.023,1.611]$ & 0.1285 \\
\hline Coronary heart disease (yes vs. no) & $1.172[0.167,8.220]$ & 0.8734 \\
\hline Hypertension (yes vs. no) & $0.662[0.080,5.501]$ & 0.7023 \\
\hline Surgery (open vs endo) & $2.882[0.468,17.725]$ & 0.2535 \\
\hline SLPI 12 h after ICU, ng/ml & $1.055[1.009,1.103]$ & 0.0181 \\
\hline
\end{tabular}

Table 5. Multivariable logistic regression model for AKI using Firth's bias correction. All patient characteristics from Table 1 with a P-value $<0.2$ in the univariable logistic regression model, the type of surgery and the SLPI measurement with the smallest P-value were taken as independent variables.

similar to other cationic peptides, such as defensins by disrupting microbial membranes ${ }^{39,40}$. By its immunomodulatory, anti-proteolytic, and anti-microbial action, SLPI functions as a regulator of innate and adaptive host defense $e^{8,41}$.

In this prospective, observational study with 33 patients undergoing open or endovascular TAAA repair, we found SLPI to be a candidate biomarker of postoperative AKI with the best predictive accuracy during the first 12 to 24 hours deeming SLPI as an early biomarker. While serum SLPI was significantly elevated in the postoperative time course on the ICU, serum SLPI levels were significantly reduced directly after surgery at the time point of admission to ICU. The half-life of serum SLPI was shown to range between 10 and 120 minutes ${ }^{42}$. Potentially, dilutions effects by perioperative volume management along with accelerated degradation of SLPI and a reduced 


\begin{tabular}{|l|l|l|l|l|l|l|}
\hline \multirow{2}{*}{$\begin{array}{l}\text { Time of } \\
\text { measurement }\end{array}$} & \multicolumn{4}{l|}{ Optimal Cut-Off (Youden index) } & \multirow{2}{*}{ AUC } \\
\cline { 2 - 7 } & Cut-Off, ng/ml & Sensitivity [\%] & Specificity [\%] & LR+ & LR- & AUC \\
\hline Baseline & $\geq 95.52$ & $11.76[1.4,36.4]$ & $100[79.4,100]$ & - & 0.88 & $0.438[0.234,0.641]$ \\
\hline Admission to ICU & $\geq 46.38$ & $57.14[28.9,82.3]$ & $91.67[61.5,99.8]$ & 6.86 & 0.47 & $0.649[0.414,0.883]$ \\
\hline 12 h after ICU & $\geq 70.03$ & $76.47[50.1,93.2]$ & $87.50[61.7,98.4]$ & 6.12 & 0.27 & $0.838[0.7,0.976]$ \\
\hline $24 \mathrm{~h}$ after ICU & $\geq 56.33$ & $75.00[47.6,92.7]$ & $71.43[41.9,91.6]$ & 2.63 & 0.35 & $0.723[0.523,0.923]$ \\
\hline $48 \mathrm{~h}$ after ICU & $\geq 61.05$ & $80.00[51.9,95.7]$ & $73.33[44.9,92.2]$ & 3.00 & 0.27 & $0.693[0.477,0.909]$ \\
\hline $72 \mathrm{~h}$ after ICU & $\geq 67.25$ & $42.86[17.7,71.1]$ & $92.31[64.0,99.8]$ & 5.57 & 0.62 & $0.648[0.432,0.865]$ \\
\hline
\end{tabular}

Table 6. Diagnostic ability of SLPI to predict AKI. ROC analysis was performed to evaluate the diagnostic ability of perioperative SLPI levels during the first $72 \mathrm{~h}$ on ICU with regard to AKI. If an elevated SLPI value indicates that the patient is likely to develop an AKI, the ROC curve should be farther from the bisecting line (Sensitivity $=1$-Specificity). Sensitivity, specificity and likelihood ratios $(\mathrm{LR}+/-$ ), are reported for the Youden optimal cut-off. 95\%-confidence intervals are shown in parentheses.
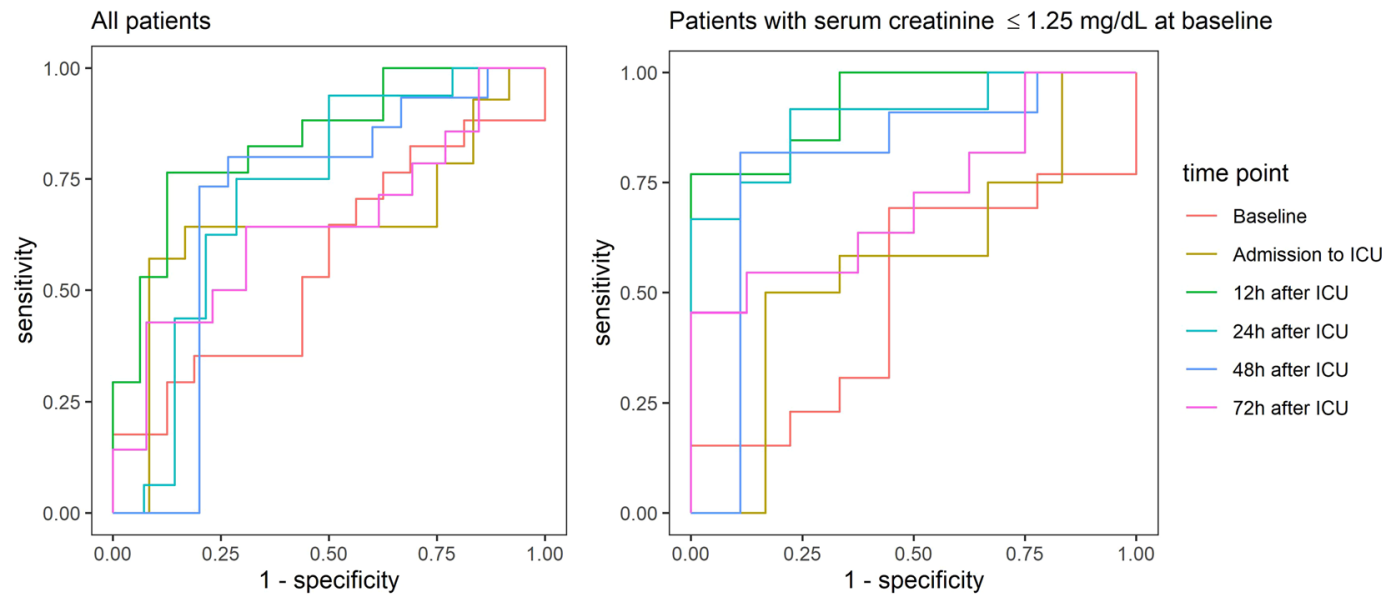

Figure 4. ROC analysis of the diagnostic accuracy of SLPI-levels for acute kidney injury in all patients and in the subgroup of patients without pre-existing renal functional impairment.

de novo synthesis during the operative procedure could contribute to the decline in serum SLPI. However, as functional data on the regulation of SLPI expression and degradation in the setting of surgical interventions are missing, to date we can only speculate on potential reasons for this observation.

Despite the different invasiveness and divergent pathophysiological mechanisms leading to AKI, there were no relevant differences in serum SLPI levels in the endovascular and open repair group. Twelve hours after complex aortic intervention, patients with AKI depicted significantly increased serum SLPI and SLPI was negatively correlated with urine output. Serum SLPI performed well to predict AKI, with a promising diagnostic accuracy of 12 and 24 hours after admission to ICU. A multivariable analysis confirmed the additional prognostic value of postoperative serum SLPI to predict AKI.

Pre-operatively increased serum creatinine $>1.25 \mathrm{mg} / \mathrm{dl}$ is one of the parameters used for perioperative risk stratification of AKI after major surgery ${ }^{43}$. As awareness regarding the occurrence of AKI might be not appropriate in those patients without pre-existing kidney function impairment, there is a special interest to elucidate the risk of AKI in patients with non-compromised preoperative renal function ${ }^{44}$. Hence, patients suffering from pre-existing renal dysfunction were excluded in an additional analysis. Interestingly, after exclusion of these patients the test accuracy significantly improved for all postoperative time points. The reason why the prognostic performance of SLPI is better in patients without chronic kidney dysfunction remains elusive. One potential explanation might be the fact, that SLPI is a protein that under physiologic conditions is efficiently degraded in tubular cells whereas in uremic patients increased plasma levels of SLPI are found which might impair the performance of SLPI as an acute biomarker of $\mathrm{AKI}^{45,46}$.

The results obtained from this observational study remain correlative and cannot explain causality. Therefore, the pathophysiological function of elevated serum SLPI needs to be discussed and investigated in different settings of cardiovascular surgery. Studies investigating the effect of SLPI during organ damage, overall establish a tissue protective role of SLPI by modulating inflammation. In an animal model, myocardial contractility was impaired in Slpi $i^{-1-}$ hearts and fully restored when SLPI was added to the preservation solution ${ }^{47}$. In the context of acute and chronic lung injury, animal models revealed a protective role of SLPI by limiting neutrophil elastase induced inflammation and anti-inflammatory, and antimicrobial activity ${ }^{48}$. Similarly, a dysregulated inflammation may be involved in the pathogenesis of AKI after TAAA repair. Hence, the extensive release of SLPI during aortic surgery may be part of the inflammatory response and a compensatory mechanism to balance the 


\begin{tabular}{|l|l|l|l|l|l|l|}
\hline \multirow{2}{*}{$\begin{array}{l}\text { Time of } \\
\text { measurement }\end{array}$} & \multicolumn{4}{|l|}{ Optimal Cut-Off (Youden index) } & \multirow{2}{*}{ AUC } \\
\cline { 2 - 7 } & Cut-Off, ng/ml & Sensitivity [\%] & Specificity [\%] & LR+ & LR- & AUC \\
\hline Baseline & $\geq 49.84$ & $69.23[38.6,90.9]$ & $55.56[21.2,86.3]$ & 1.56 & 0.55 & $0.496[0.235,0.756]$ \\
\hline Admission to ICU & $\geq 49.67$ & $50.00[21.1,78.9]$ & $83.33[35.9,99.6]$ & 3.00 & 0.60 & $0.569[0.253,0.886]$ \\
\hline 12 h after ICU & $\geq 57.02$ & $76.92[46.2,95.0]$ & $100[66.4,100]$ & - & 0.23 & $0.932[0.832,1]$ \\
\hline 24h after ICU & $\geq 43.20$ & $91.67[61.5,99.8]$ & $77.78[40.0,97.2]$ & 4.13 & 0.11 & $0.898[0.763,1]$ \\
\hline 48h after ICU & $\geq 71.24$ & $81.82[48.2,97.7]$ & $88.89[51.8,99.7]$ & 7.36 & 0.20 & $0.798[0.560,1]$ \\
\hline 72 h after ICU & $\geq 68.81$ & $45.45[16.7,76.6]$ & $100[63.1,100]$ & - & 0.55 & $0.716[0.477,0.955]$ \\
\hline
\end{tabular}

Table 7. Diagnostic ability of SLPI to predict AKI in the subgroup of patients with serum creatinine at baseline $\leq 1.25 \mathrm{mg} / \mathrm{dL}$. ROC analysis was performed to evaluate the diagnostic ability of perioperative SLPI levels during the first $72 \mathrm{~h}$ on ICU with regard to AKI. If an elevated SLPI value indicates that the patient is likely to develop an AKI, the ROC curve should be farther from the bisecting line (Sensitivity = 1-Specificity). Sensitivity, specificity and likelihood ratios (LR+/-), are reported for the Youden optimal cut-off. 95\%-confidence intervals are shown in parentheses.

inflammatory reaction ${ }^{47}$. This hypothesis is supported by our observation of a significant correlation between serum SLPI and procalcitonin, an early inflammatory marker of the immune response, 24 and 72 hours after surgery.

Although, SLPI was suggested to exert kidney protection via promoting tubular cell regeneration, data on the functional role of SLPI in AKI and in critically ill patients are scarce ${ }^{13}$. Thus, experimental studies elucidating the pathophysiological effects of SLPI on oxidative stress and kidney injury are needed. Of note, the assumed protective role of SLPI during organ dysfunction could potentially be exploited therapeutically by mimicking SLPI's organ protective functions.

Regarding potential limitations of this study, the following aspects need to be mentioned: As only few patients suffering from TAAA are treated by open or endovascular means annually world-wide, only few patients could be included in this study. Furthermore, it would have been favorable to include only patients treated by open or endovascular modality. As for most observational studies, investigating the diagnostic accuracy of biomarkers of AKI, another limitation is that kidney biopsies are not routinely available for the diagnosis of AKI based on histopathological tubular injury ("gold standard"). Yet, the diagnosis is commonly based on the two parameters serum creatinine and urine output, which are lacking sensitivity and specificity for the detection of kidney tubular injury. In the future, this general restriction will potentially be resolved by the identification of damage associated AKI biomarkers as the new gold standards of AKI. Even if the results of our study are promising and the test quality is good, the hypothesis-generating character of this study needs to be emphasized: The results should be validated by follow-up clinical studies to verify the clinical significance of SLPI as a promising new biomarker of acute kidney failure and other severe complications after major surgical interventions.

\section{Conclusion}

The presented results highlight SLPI as a promising, new biomarker for the detection of postoperative acute kidney after open and endovascular TAAA repair within 72 hours, which may enable the early initiation of organ-protective therapy and reduce the incidence and sequela of AKI and other postoperative complications.

Contribution to the field. Thoracoabdominal aortic aneurysm (TAAA) repair is related to a relevant rate of postoperative complications including acute kidney injury (AKI) with the highest incidence, which are closely associated with outcome. Up to now, post-operative AKI detection is mainly based on urine output and serum creatinine levels. Even if serum creatinine is an established biomarker, due to its delayed increase and low sensitivity for the detection of an impaired kidney function a critical assessment is required. Any optimization of the peri-operative and post-operative surveillance could lead to a better understanding of the complex inflammatory processes, which are activated by the required surgical trauma. Biomarkers, such as Secretory leucocyte peptidase inhibitor could enable an earlier detection of severe organ dysfunction, leading to a more appropriate and especially faster diagnosis and therapy. As indicated by our findings, a standardized usage of biomarkers for early detection of organ failure after major surgery such as TAAA repair may improve patients' outcome.

\section{Data availability}

All datasets supporting the findings of this study are included within the manuscript or its supplemental data files.

Received: 18 October 2019; Accepted: 13 February 2020;

Published online: 26 February 2020

\section{References}

1. Thakar, C. V. Perioperative acute kidney injury. Adv. Chronic Kidney Dis. 20, 67-75, https://doi.org/10.1053/j.ackd.2012.10.003 (2013).

2. Chertow, G. M., Burdick, E., Honour, M., Bonventre, J. V. \& Bates, D. W. Acute kidney injury, mortality, length of stay, and costs in hospitalized patients. J. Am. Soc. Nephrology: JASN 16, 3365-3370, https://doi.org/10.1681/ASN.2004090740 (2005).

3. Saratzis, A., Melas, N., Mahmood, A. \& Sarafidis, P. Incidence of Acute Kidney Injury (AKI) after Endovascular Abdominal Aortic Aneurysm Repair (EVAR) and Impact on Outcome. Eur. J. Vasc. Endovasc. Surg. 49, 534-540, https://doi.org/10.1016/j. ejvs.2015.01.002 (2015) 
4. Nickolas, T. L. et al. Diagnostic and prognostic stratification in the emergency department using urinary biomarkers of nephron damage: a multicenter prospective cohort study. J. Am. Coll. Cardiol. 59, 246-255, https://doi.org/10.1016/j.jacc.2011.10.854 (2012).

5. Moran, S. M. \& Myers, B. D. Course of acute renal failure studied by a model of creatinine kinetics. Kidney Int. 27, 928-937, https:// doi.org/10.1038/ki.1985.101 (1985)

6. Kellum, J. A. \& Prowle, J. R. Paradigms of acute kidney injury in the intensive care setting. Nat. Rev. Nephrol. 14, 217-230, https:// doi.org/10.1038/nrneph.2017.184 (2018).

7. Thompson, R. C. \& Ohlsson, K. Isolation, properties, and complete amino acid sequence of human secretory leukocyte protease inhibitor, a potent inhibitor of leukocyte elastase. Proc. Natl Acad. Sci. U S Am. 83, 6692-6696 (1986).

8. Majchrzak-Gorecka, M., Majewski, P., Grygier, B., Murzyn, K. \& Cichy, J. Secretory leukocyte protease inhibitor (SLPI), a multifunctional protein in the host defense response. Cytokine growth factor. Rev. 28, 79-93, https://doi.org/10.1016/j. cytogfr.2015.12.001 (2016).

9. Sallenave, J. M. et al. Secretory leukocyte proteinase inhibitor is preferentially increased in patients with acute respiratory distress syndrome. Eur. respiratory J. 13, 1029-1036 (1999).

10. Ilzecka, J. \& Stelmasiak, Z. Increased serum levels of endogenous protectant secretory leukocyte protease inhibitor in acute ischemic stroke patients. Cerebrovasc. Dis. 13, 38-42, https://doi.org/10.1159/000047744 (2002).

11. Nernpermpisooth, N., Prompunt, E. \& Kumphune, S. An in vitro endothelial cell protective effect of secretory leukocyte protease inhibitor against simulated ischaemia/reperfusion injury. Exp. therapeutic Med. 14, 5793-5800, https://doi.org/10.3892/ etm.2017.5272 (2017).

12. Ghasemlou, N. et al. Beneficial effects of secretory leukocyte protease inhibitor after spinal cord injury. Brain: a J. Neurol. 133, 126-138, https://doi.org/10.1093/brain/awp304 (2010).

13. Ochi, A. et al. MIF-2/D-DT enhances proximal tubular cell regeneration through SLPI- and ATF4-dependent mechanisms. Am. J. Physiol. Ren. Physiol 313, F767-F780, https://doi.org/10.1152/ajprenal.00683.2016 (2017).

14. Wilflingseder, J. et al. Molecular pathogenesis of post-transplant acute kidney injury: assessment of whole-genome mRNA and miRNA profiles. PLoS one 9, e104164, https://doi.org/10.1371/journal.pone.0104164 (2014).

15. Averdunk, L. et al. Secretory Leukocyte Protease Inhibitor (SLPI)-A Novel Predictive Biomarker of Acute Kidney Injury after Cardiac Surgery: A Prospective Observational Study. J Clin Med 8, https://doi.org/10.3390/jcm8111931 (2019).

16. Crawford, E. S. Thoraco-abdominal and abdominal aortic aneurysms involving renal, superior mesenteric, celiac arteries. Ann. Surg. 179, 763-772 (1974).

17. Lamb, E. J., Levey, A. S. \& Stevens, P. E. The Kidney Disease Improving Global Outcomes (KDIGO) guideline update for chronic kidney disease: evolution not revolution. Clin. Chem. 59, 462-465, https://doi.org/10.1373/clinchem.2012.184259 (2013).

18. Mommertz, G. et al. Thoracoabdominal aortic aneurysm repair in patients with marfan syndrome. Eur. J. Vasc. Endovasc. surgery: Off. J. Eur. Soc. Vasc. Surg. 35, 181-186, https://doi.org/10.1016/j.ejvs.2007.10.013 (2008).

19. Jacobs, M. J. \& Schurink, G. W. Open repair in chronic type B dissection with connective tissue disorders. Ann. Cardiothorac. Surg. 3, 325-328, https://doi.org/10.3978/j.issn.2225-319X.2014.05.02 (2014)

20. Jacobs, M. J., Elenbaas, T. W., Schurink, G. W., Mess, W. H. \& Mochtar, B. Assessment of spinal cord integrity during thoracoabdominal aortic aneurysm repair. The Annals of thoracic surgery 74, S1864-1866; discussion S1892-1868 (2002).

21. Tshomba, Y. et al. Open treatment of extent IV thoracoabdominal aortic aneurysms. J. cardiovascular Surg. 56, 687-697 (2015).

22. Canyigit, M. et al. Reduction of iodinated contrast load with the renal artery catheterization technique during endovascular aortic repair. Diagnostic interventional radiology 19, 244-250, https://doi.org/10.5152/dir.2012.001 (2013).

23. Thiele, R. H., Isbell, J. M. \& Rosner, M. H. AKI associated with cardiac surgery. Clin. J. Am. Soc. Nephrol. 10, 500-514, https://doi. org/10.2215/CJN.07830814 (2015).

24. American Thoracic, S., Infectious Diseases Society of, A. Guidelines for the management of adults with hospital-acquired, ventilatorassociated, and healthcare-associated pneumonia. Am. J. Respir. Crit. Care Med. 171, 388-416, https://doi.org/10.1164/rccm.200405644ST (2005).

25. De Leyn, P. et al. Tracheotomy: clinical review and guidelines. Eur. J. Cardiothorac. Surg. 32, 412-421, https://doi.org/10.1016/j. ejcts.2007.05.018 (2007).

26. Kurmani, S. \& Squire, I. Acute Heart Failure: Definition, Classification and Epidemiology. Curr. Heart Fail. Rep. 14, 385-392, https:// doi.org/10.1007/s11897-017-0351-y (2017).

27. Asaria, P. et al. Acute myocardial infarction hospital admissions and deaths in England: a national follow-back and follow-forward record-linkage study. Lancet Public. Health 2, e191-e201, https://doi.org/10.1016/S2468-2667(17)30032-4 (2017).

28. Weissler-Snir, A., Adler, A., Williams, L., Gruner, C. \& Rakowski, H. Prevention of sudden death in hypertrophic cardiomyopathy: bridging the gaps in knowledge. Eur. Heart J. 38, 1728-1737, https://doi.org/10.1093/eurheartj/ehw268 (2017).

29. Vincent, J. L., Opal, S. M., Marshall, J. C. \& Tracey, K. J. Sepsis definitions: time for change. Lancet 381, 774-775, https://doi. org/10.1016/S0140-6736(12)61815-7 (2013).

30. Team, R. C. in R Foundation for Statistical Computing, Vienna, Austria (2014).

31. Spanos, K. et al. Risk of spinal cord ischemia after fenestrated or branched endovascular repair of complex aortic aneurysms. J. Vasc. Surg. 69, 357-366, https://doi.org/10.1016/j.jvs.2018.05.216 (2019).

32. Gombert, A. et al. Outcomes After One Stage Versus Two Stage Open Repair of Type II Thoraco-abdominal Aortic Aneurysms. European journal of vascular and endovascular surgery: the official journal of the European Society for Vascular Surgery, https://doi. org/10.1016/j.ejvs.2018.09.007 (2018).

33. Meersch, M. et al. Prevention of cardiac surgery-associated AKI by implementing the KDIGO guidelines in high risk patients identified by biomarkers: the PrevAKI randomized controlled trial. Intensive Care Med. 43, 1551-1561, https://doi.org/10.1007/ s00134-016-4670-3 (2017).

34. Zarbock, A. et al. Effect of Early vs Delayed Initiation of Renal Replacement Therapy on Mortality in Critically Ill Patients With Acute Kidney Injury: The ELAIN Randomized Clinical Trial. JAMA 315, 2190-2199, https://doi.org/10.1001/jama.2016.5828 (2016).

35. Gombert, A. et al. Urine neutrophil gelatinase-associated lipocalin predicts outcome and renal failure in open and endovascular thoracic abdominal aortic aneurysm surgery. Sci. Rep. 8, 12676, https://doi.org/10.1038/s41598-018-31183-1 (2018).

36. Camper, N. et al. A secretory leukocyte protease inhibitor variant with improved activity against lung infection. Mucosal immunology 9, 669-676, https://doi.org/10.1038/mi.2015.90 (2016)

37. Wright, C. D., Kennedy, J. A., Zitnik, R. J. \& Kashem, M. A. Inhibition of murine neutrophil serine proteinases by human and murine secretory leukocyte protease inhibitor. Biochemical biophysical Res. Commun. 254, 614-617, https://doi.org/10.1006/bbrc.1998.0108 (1999).

38. Taggart, C. C., Greene, C. M., McElvaney, N. G. \& O’Neill, S. Secretory leucoprotease inhibitor prevents lipopolysaccharide-induced IkappaBalpha degradation without affecting phosphorylation or ubiquitination. J. Biol. Chem. 277, 33648-33653, https://doi. org/10.1074/jbc.M203710200 (2002).

39. Verma, C. et al. Defensins: antimicrobial peptides for therapeutic development. Biotechnol. J. 2, 1353-1359, https://doi.org/10.1002/ biot.200700148 (2007).

40. Tomee, J. F., Hiemstra, P. S., Heinzel-Wieland, R. \& Kauffman, H. F. Antileukoprotease: an endogenous protein in the innate mucosal defense against fungi. J. Infect. Dis. 176, 740-747 (1997). 
41. Ohlsson, S., Ljungkrantz, I., Ohlsson, K., Segelmark, M. \& Wieslander, J. Novel distribution of the secretory leucocyte proteinase inhibitor in kidney. Mediators Inflamm. 10, 347-350, https://doi.org/10.1080/09629350120102389 (2001).

42. Bergenfeldt, M., Bjork, P. \& Ohlsson, K. The elimination of secretory leukocyte protease inhibitor (SLPI) after intravenous injection in dog and man. Scand. J. Clin. Lab. Invest. 50, 729-737, https://doi.org/10.1080/00365519009091066 (1990).

43. Thakar, C. V., Arrigain, S., Worley, S., Yared, J. P. \& Paganini, E. P. A clinical score to predict acute renal failure after cardiac surgery. J. Am. Soc. Nephrol. 16, 162-168, https://doi.org/10.1681/ASN.2004040331 (2005).

44. Singh, P., Rifkin, D. E. \& Blantz, R. C. Chronic kidney disease: an inherent risk factor for acute kidney injury? Clin. J. Am. Soc. Nephrol. 5, 1690-1695, https://doi.org/10.2215/CJN.00830110 (2010).

45. Nystrom, M., Bergenfeldt, M. \& Ohlsson, K. The elimination of secretory leukocyte protease inhibitor (SLPI) from the gastrointestinal tract in man. Scand. J. Clin. Lab. Invest. 57, 119-125 (1997).

46. Strober, W. \& Waldmann, T. A. The role of the kidney in the metabolism of plasma proteins. Nephron 13, 35-66, https://doi. org/10.1159/000180368 (1974).

47. Schneeberger, S. et al. The effect of secretory leukocyte protease inhibitor (SLPI) on ischemia/reperfusion injury in cardiac transplantation. Am. J. transplantation: Off. J. Am. Soc. Transpl. Am. Soc. Transplant Surg. 8, 773-782, https://doi. org/10.1111/j.1600-6143.2008.02158.x (2008).

48. Nishimura, J. et al. Potent antimycobacterial activity of mouse secretory leukocyte protease inhibitor. J. immunology 180, 4032-4039 (2008).

\section{Acknowledgements}

This research received no external funding.

\section{Author contributions}

Conceptualization, A.G., L.A., G.M. and C.S.; Formal analysis, M.R., L.A., A.G.; Investigation L.A., Methodology, A.G., C.S., M.R.; Writing - original draft, A.G., L.A., M.R., C.S.; Writing - review \& editing: A.G., L.A., M.R., A.Z., H.J., M.J., L.M.

\section{Competing interests}

The authors declare that they have no competing interests. Furthermore the authors declare that the research was conducted in the absence of any commercial or financial relationships that could be construed as a potential conflict of interest.

\section{Additional information}

Supplementary information is available for this paper at https://doi.org/10.1038/s41598-020-60482-9.

Correspondence and requests for materials should be addressed to A.G.

Reprints and permissions information is available at www.nature.com/reprints.

Publisher's note Springer Nature remains neutral with regard to jurisdictional claims in published maps and institutional affiliations.

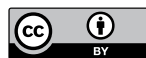

Open Access This article is licensed under a Creative Commons Attribution 4.0 International License, which permits use, sharing, adaptation, distribution and reproduction in any medium or format, as long as you give appropriate credit to the original author(s) and the source, provide a link to the Creative Commons license, and indicate if changes were made. The images or other third party material in this article are included in the article's Creative Commons license, unless indicated otherwise in a credit line to the material. If material is not included in the article's Creative Commons license and your intended use is not permitted by statutory regulation or exceeds the permitted use, you will need to obtain permission directly from the copyright holder. To view a copy of this license, visit http://creativecommons.org/licenses/by/4.0/.

(C) The Author(s) 2020 